\title{
SWARM ALGORITHM BASED ADAPTIVE FILTER DESIGN TO REMOVE POWER LINE INTERFERENCE FROM ECG SIGNAL
}

\author{
Vikram B. Galphade ${ }^{1}$, P. C. Bhaskar ${ }^{2}$ \\ ${ }^{I}$ Electronics Department, Department of Technology, Shivaji University, Kolhapur, India (MS) \\ ${ }^{2}$ Electronics Department, Department of Technology, Shivaji University, Kolhapur, India (MS)
}

\begin{abstract}
ECG signal is having wide importance in the biomedical field, but for proper diagnosis of ECG always a noise free ECG signal is needed. Many researchers have already developed filters for getting appropriate desirable ECG signal and till today many researchers are still developing different filters using different algorithms in order to get clearer ECG signal for proper diagnosis. Noises and Interferences get added in the ECG by different ways, at the time of ECG Acquisition or at the time of ECG signal recording.

In this paper newly adapted algorithm is used for the filtering of ECG signal that is a Swarm algorithm which is used for the Error signal optimization from the original corrupted ECG signal. This algorithm is implemented with Adaptive filter to removes Power Line Interference noise having Frequency component of $50 \mathrm{~Hz}$. The ECG signal considered may be retrieved from ECG acquisition system or from MIT-BIH database.
\end{abstract}

Keywords: Adaptive Filter, SWARM Algorithm, MIT-BIH Database, Matlab, ECG Signal and Power line Noise Signal etc.

\section{INTRODUCTION}

Electrocardiogram (ECG) signal is very important in the biomedical field for diagnosis of diseases related to heart. Heart's electrical pulses is measured by ECG signal. It captures electrical impulse generated by the polarization and depolarization of cardiac tissue and represent in the form of wave [5]. ECG signal contains P, Q, R, S and T different intervals and segments which represents different conditions of heart.

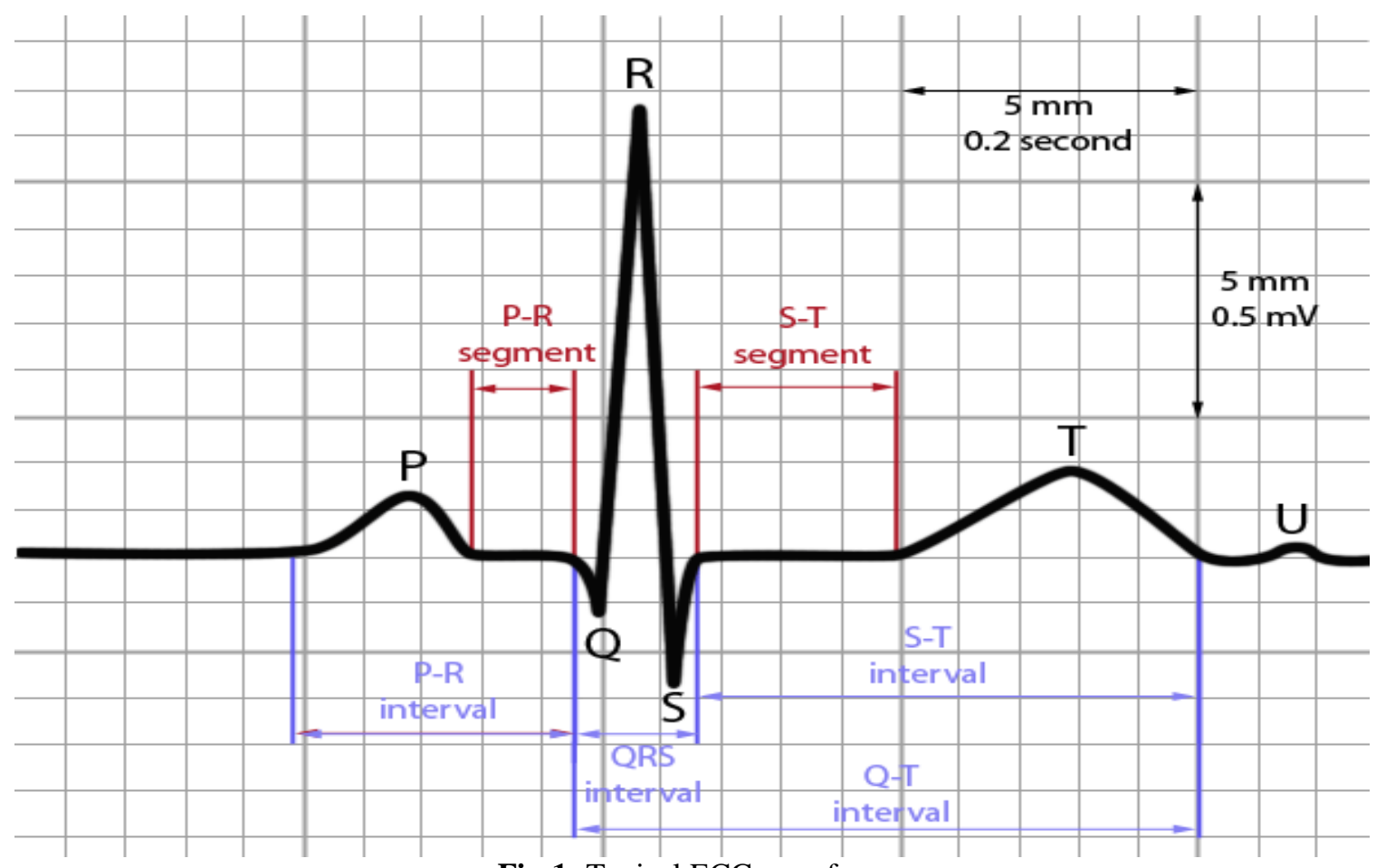

Fig-1: Typical ECG waveform 
Electrocardiogram (ECG) signal is corrupted due to addition of different noises or interferences, out of that noises Power Line Noise is more effective than other interferences [10]. Power Line Noise of $50 \mathrm{~Hz}$ or $60 \mathrm{~Hz}$ and amplitude varies from $5 \%$ to $10 \%$ of peak amplitude of ECG signal.

This $50 \mathrm{~Hz}$ or $60 \mathrm{~Hz}$ frequency component destroy low frequency component. Harmonics present, in the Power Line Noise decreases the quality of the ECG signal and affect the tiny features. ECG signal having frequency range of $0 \mathrm{~Hz}$ to $150 \mathrm{~Hz}$ typically. This will affect QRS complex [8] and ST segment [7] of ECG signal, which is important for clinical diagnosis, monitoring and signal processing. Hence it is necessary to remove Power Line Interference Noise from the ECG signal. There are different methods to remove noise from the ECG signal like FIR equi-ripple notch filter, IIR notch filter and discrete wavelet transformation etc. These methods requires more stages and consumes more power for processing.

Adaptive filters effectively removes noise from the ECG signal with less power consumption, because it requires less no of stages compared to FIR and IIR filters. Adaptive filter with swarm algorithm is newly developed technique. Swarm algorithm is based on the principle of collective behavior of result and it is studied and verified for ECG signal.

\section{PROPOSED SYSTEM}

\subsection{ECG Signal Extraction}

Electrocardiogram (ECG) signal required, is extracted from MIT-BIH (Massachusetts Institute of Technology/ Beth Israel Hospital) database [10]. MIT-BIH database used for the ECG signal extraction due to following reasons:

1. MIT-BIH database contain recording of 30 minutes of each patients, which is sufficient longer than other database

2. It contains records of the normal ECG signal.

MIT-BIH database contain variety of recorded samples, which is used for the clinical practice. It contains 48 annotated records. This records obtained from 47 subject by studies of arrhythmia laboratory of Beth Israel Hospital in Boston 1975 to 1979. MIT-BIH Arrhythmia database considerably variations in the signal quality with significant portion with significant portion of unreadable data in at least one lead. ECG signal is extracted from MIT-BIH database through MATLAB code.

\subsection{Particle Swarm Optimization Algorithm: -}

Swarm optimization algorithm is optimization mathematical technique that concerns the finding of maxima or minima of function. Particle Swarm Optimization (PSO) is relatively new modern and powerful method of optimization. It is widely used for the Global optimization solution [11].

Particle Swarm Optimization (PSO) algorithm is implemented with the Adaptive filter for minimization of the error signal from the noisy ECG signal. Implementation of the Particle Swarm Optimization algorithm with Adaptive filter is shown below
Paragraph comes content here. Paragraph comes content here. Paragraph comes content here. Paragraph comes content here. Paragraph comes content here. Paragraph comes content here. Paragraph comes content here. Paragraph comes content here.

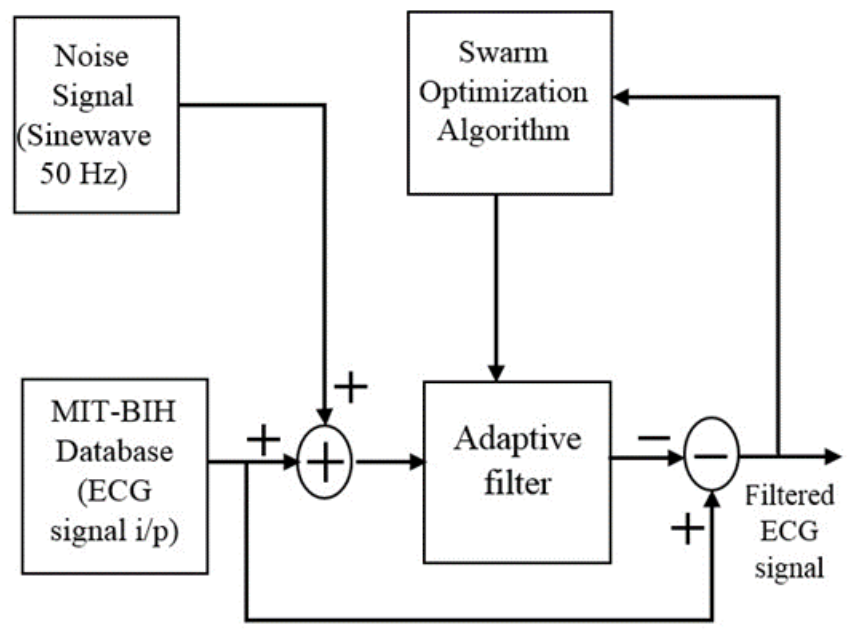

Fig-2: Block diagram of Swarm algorithm based Adaptive filter

Particle Swarm Optimization (PSO) algorithm first introduced by Dr. Kennedy and Dr. Eberhart in 1995. PSO Method is becoming more popular for its simplicity of implementation. It also has ability to swiftly converge to a good solution. The main equation for the optimization of the noise signal as given below:

Equation for the Velocity updation:

$$
\begin{gathered}
\operatorname{Vin}(\mathrm{n}+1)=\operatorname{Vin}(\mathrm{n})+\mathrm{C} 1 * \mathrm{R} 1 *(\text { Gbest }-\mathrm{X}(\mathrm{n}))+\mathrm{C} 2 * \\
\mathrm{R} 2 *(\text { Gbest }-\mathrm{X}(\mathrm{n}))
\end{gathered}
$$

Vin $(\mathrm{n})=$ Initial condition of the signal.

$\mathrm{C} 1, \mathrm{R} 1, \mathrm{C} 2$ and $\mathrm{R} 2=$ Constants of values between 0 to 1 .

Gbest = Error signal.

$\mathrm{X}(\mathrm{n})=$ Initial input considered as Zero.

Equation for the Position updation:

$$
\operatorname{Pin}(n+1)=\operatorname{Pin}(n)+\operatorname{Vin}(n)
$$

Pin $(n+1)=$ Coefficient of Adaptive filter.

The value of Vin $(n+1)$ of Eq-(1) is put in the Eq-(2) at Vin (n), for updating the value of Vin (n). Different values of Pin $(n+1)$ acting as a coefficients for different stages. These value of the Pin $(n+1)$ are changes automatically, which is depends on the value of the present input signal.

The operation of the Swarm Optimization algorithm with Adaptive filter is given below. 


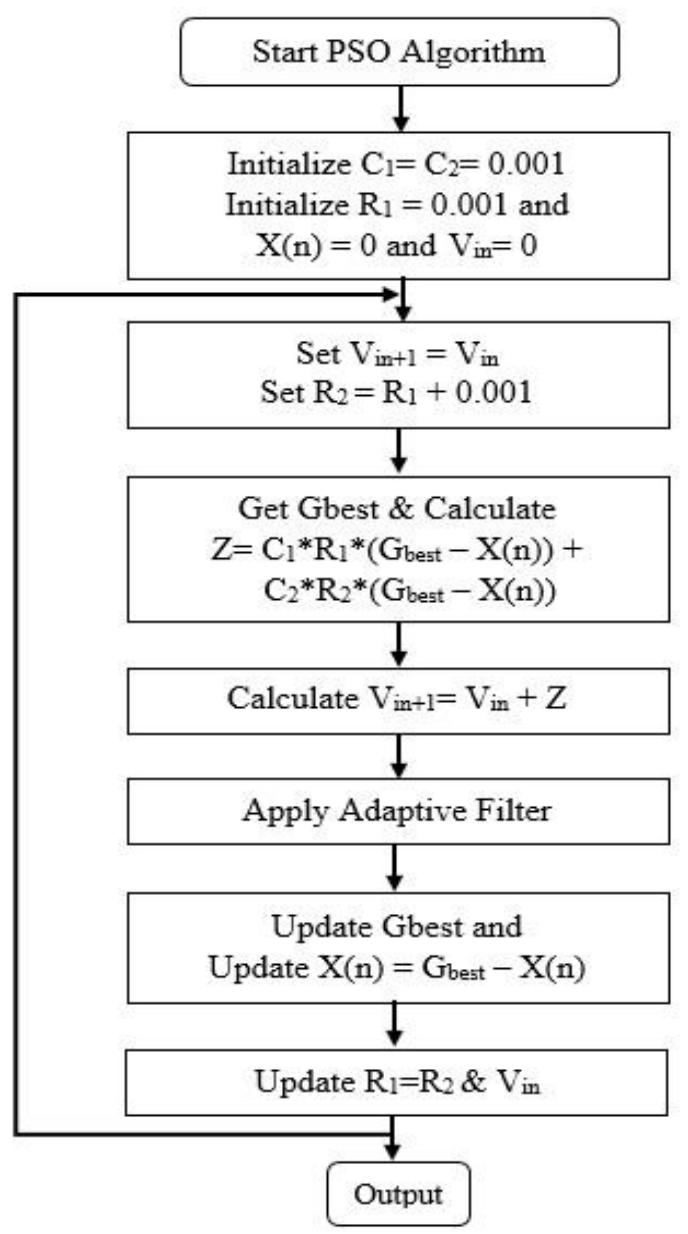

Fig-3: Operation of Swarm Optimization Algorithm based Adaptive filter

Values of the $\mathrm{C} 1, \mathrm{C} 2, \mathrm{R} 1$ and $\mathrm{R} 2$ is take in the range of the 0.001 , because amplitude range of the ECG signal is in the range of $\mathrm{mV}$.

Adaptive filer basic equation:

$$
Y(x)=\sum_{n=0}^{\infty} P(n) * X(k-n) \rrbracket \quad \text { Eq-(3) }
$$

This is the basic equation of Adaptive filter. This updated value of Pin $(n+1)$ is put in the Eq-3. In the Eq-(3) P (n) acting as a coefficient of the Adaptive filter and $X(k-n)$ is nothing but the noisy ECG signal input which we want to filter. The output of the Adaptive filter is taken as feedback signal for calculating error signal. This error signal is subtracted from the desired signal and we get filtered ECG signal at the output.

\section{DESIGN AND IMPLEMENTATION}

Swarm optimization algorithm based Adaptive filter is implemented using Matlab software version-2013b. For hardware implementation we required System generator tool and Xilinx blocks sets, hence we need Xilinx software which is compatible with the Matlab software. Xilinx 14.5 System generator tool has been used. For implementation of Swarm algorithm based Adaptive filter it is necessary minimum 6 adders, 4 multipliers and 2 constant multiplier. Depending upon this requirement and considering low cost Spartan -3 a board is selected for the hardware co-simulation.

Matlab software is very effective tool for the Simulink of Digital Signal processing. It provides variety of signal processing tools also it provides Xilinx block set for the implementation of Simulink model on hardware. System generator tool is used for the creating the JTAG block, this block is required for the burning Simulink model on the Sparten-3 board.

ECG signal retrieved from MIT-BIH database added with Power Line Interference Noise i.e. sinusoidal wave of frequency of $50 \mathrm{~Hz}$ or $60 \mathrm{~Hz}$ and gives this noisy ECG signal as input to the Adaptive filter.

Adaptive filter based on the Swarm Optimization Algorithm, which is sufficient for removing Power Line Noise from the ECG signal at single stage. Simulink model of the Swarm based Adaptive filter with single stage is given below as shown in the Fig-(4).

Hardware is connected to the computer by using JTAG cable and generate JTAG by using System generator block of the Xilinx block set. First run MIT-BIH code and get pure ECG signal from the database. Then take inputs to the JTAG and run hardware co-simulation model. Output taken from JTAG cable is hardware cosimulation of Spartan-3a board.

The subsystem shown in fig-(5) is internal structure of single stage Adaptive filter based on Swarm Optimization Algorithm shown below. In this subsystem of Swarm Optimization Algorithm is inter faced to the Adaptive filter. 


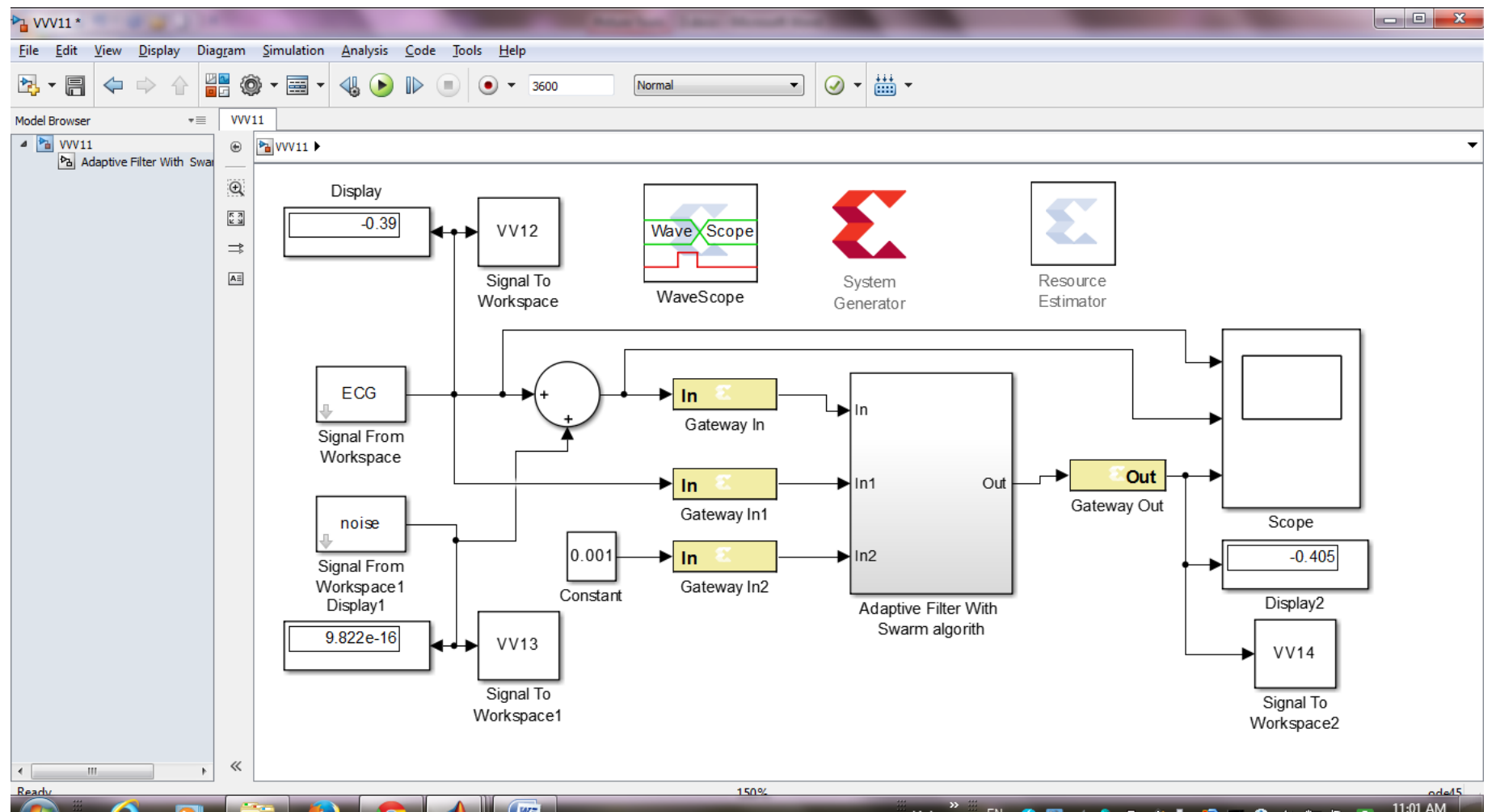

Fig-(4) Simulink model of Adaptive filter based on Swarm Optimization Algorithm.

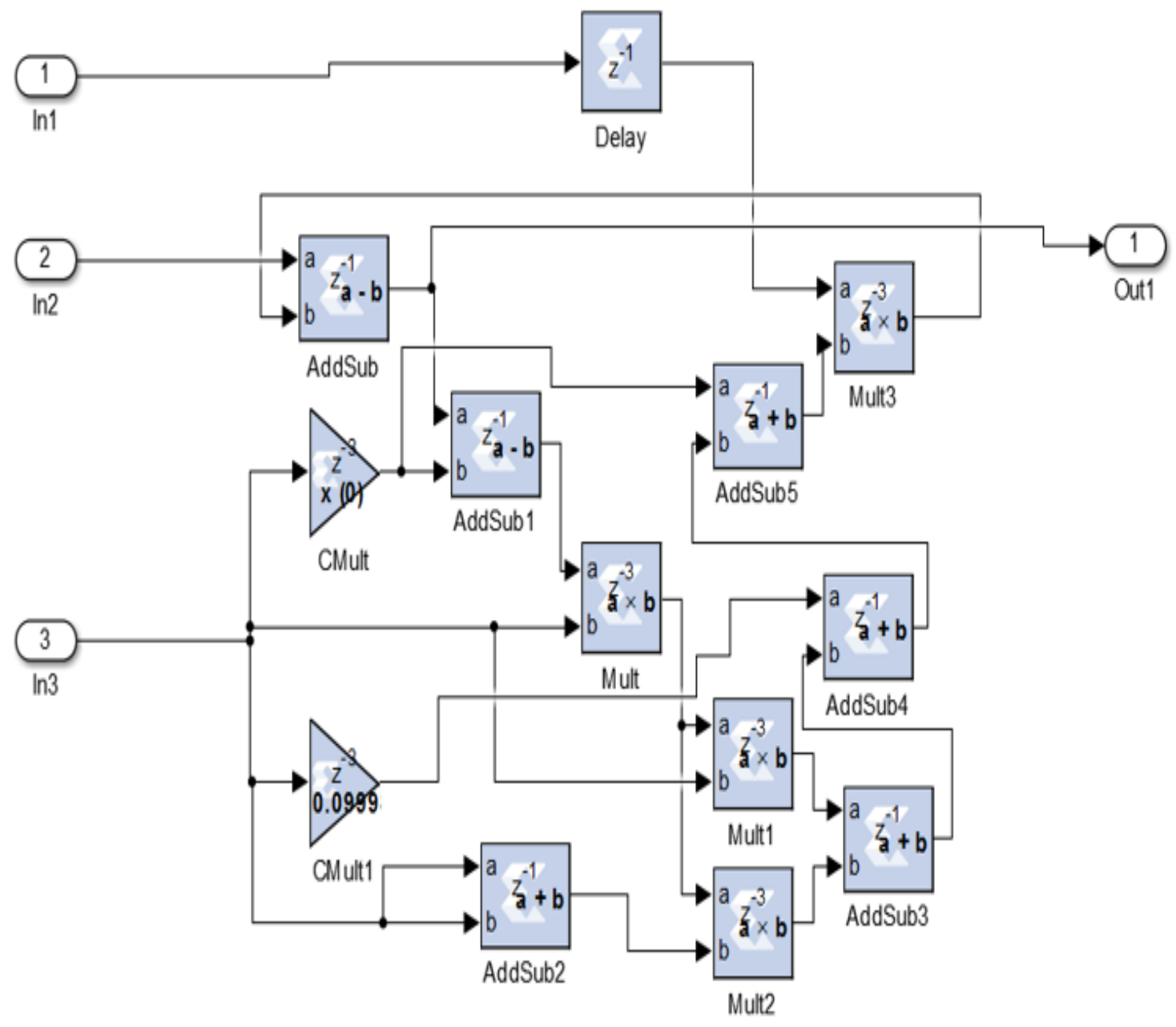

Fig-(5) Internal structure of the Swarm optimization based Adaptive filter 


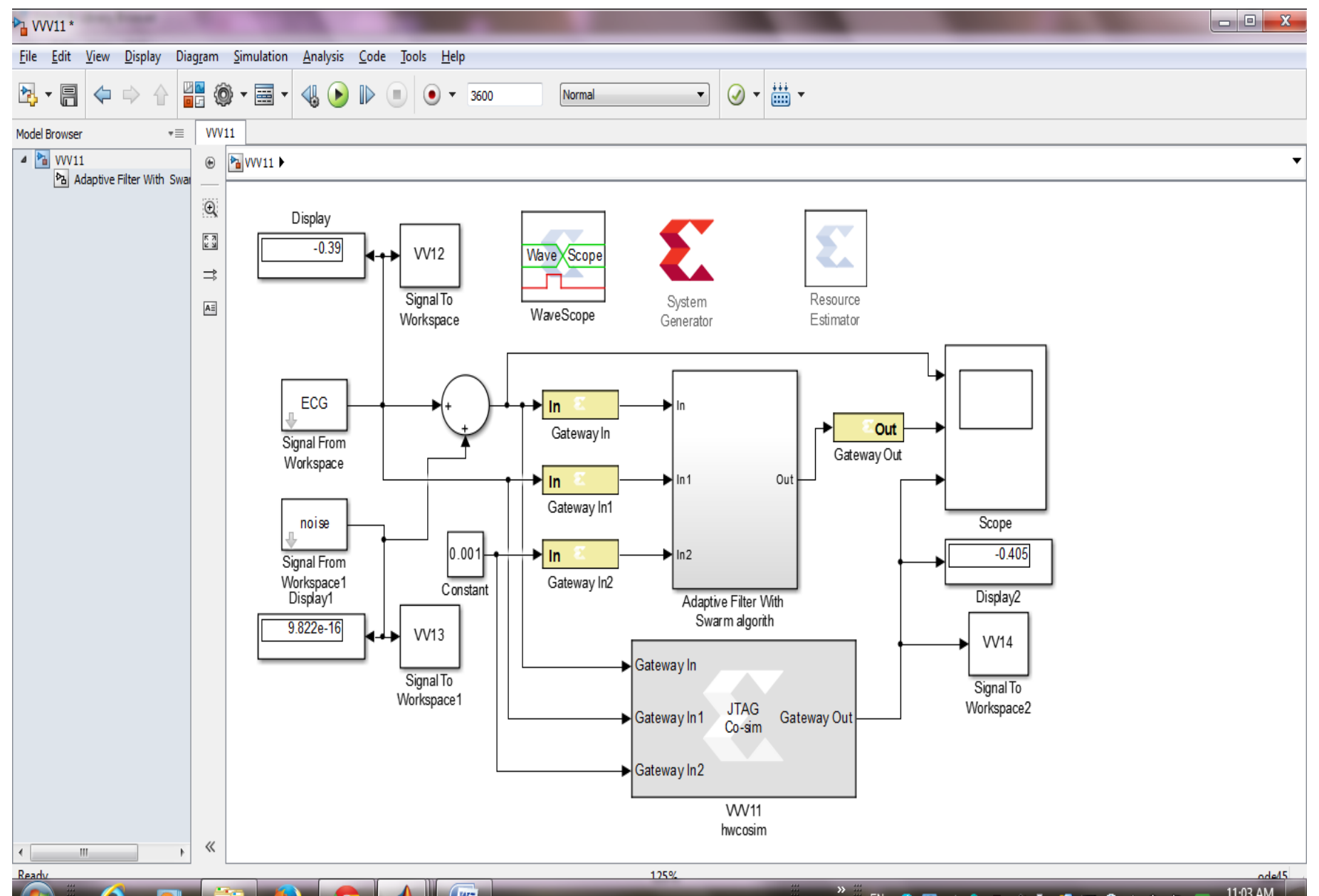

Fig-6: Hardware Co-simulation model of Adaptive filter based on Swarm Optimization Algorithm.

Hardware cosimulation model of Swarm Optimization based adaptive filter with single stage is shown in figer-(6). JTAG block generated by the system generator is connect as shown figer-(6).

\section{RESULTS}

MIT-BIH database is used for the extracting ECG signal, from that we get noise free ECG signal. This noise free ECG signal is used as an desired signal input to Swarm Optimization Algorithm and by adding sinusoidal of $50 \mathrm{~Hz}$ resultant signal is used as input to Adaptive filter.

Matlab Simulink model of single stage Adaptive filter based on Swarm algorithm. This designed single stage Adaptive filter is sufficient to remove Power Line Noise from ECG signal.

Simulink model result of single stage Adaptive filter is very clear and sharp as shown in Fig-(7) below, hence it is not necessary to go through more stages.

JTAG cable connect to the Spartan-3 board and generate JTAG for given input signals as shown in Fig-(6). Result obtained from the hardware cosimulation model is shown in Fig-(8). Fig-(9) shows result of wavescope, which gives values of signal at different positions.
Result from the single stage Adaptive filter is same as that of the multistage Adaptive filter. Hence it does not required more stages for removing Power Line Noise from the ECG signal. Result of 2 nd and 3rd stage swarm based Adaptive filter is shown in fig-(10) and fig-(11). Adaptive filter with single stage required less power and time for filtering ECG signal. 
Scope

回

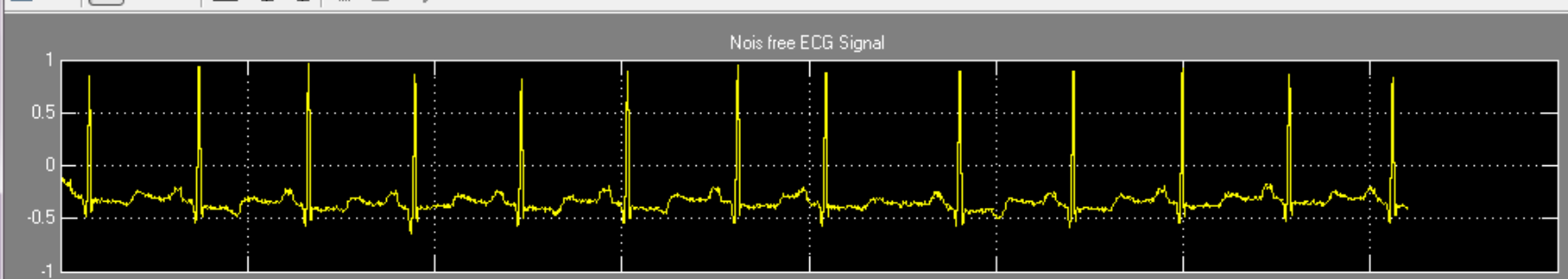

\section{Noisy Filtered ECG Signal}

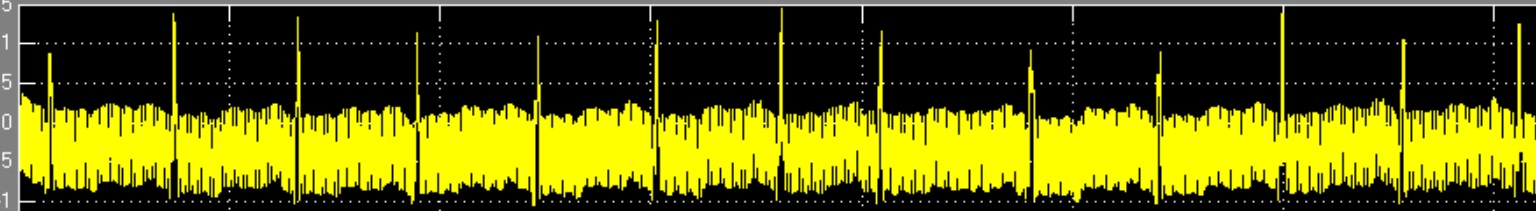
$-1.5$

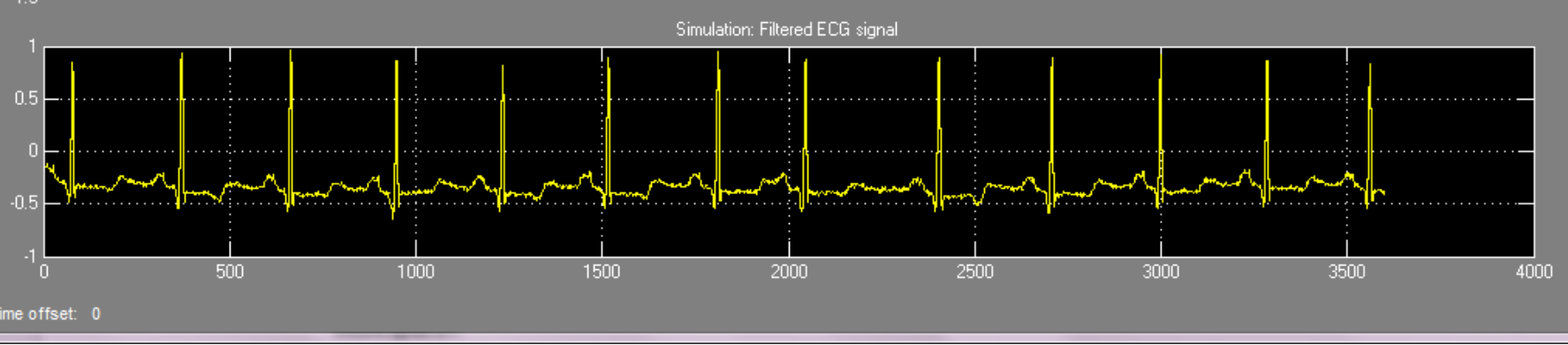

Fig-7: Simulation result of Filtered ECG signal of Swarm algorithm based Adaptive filter.

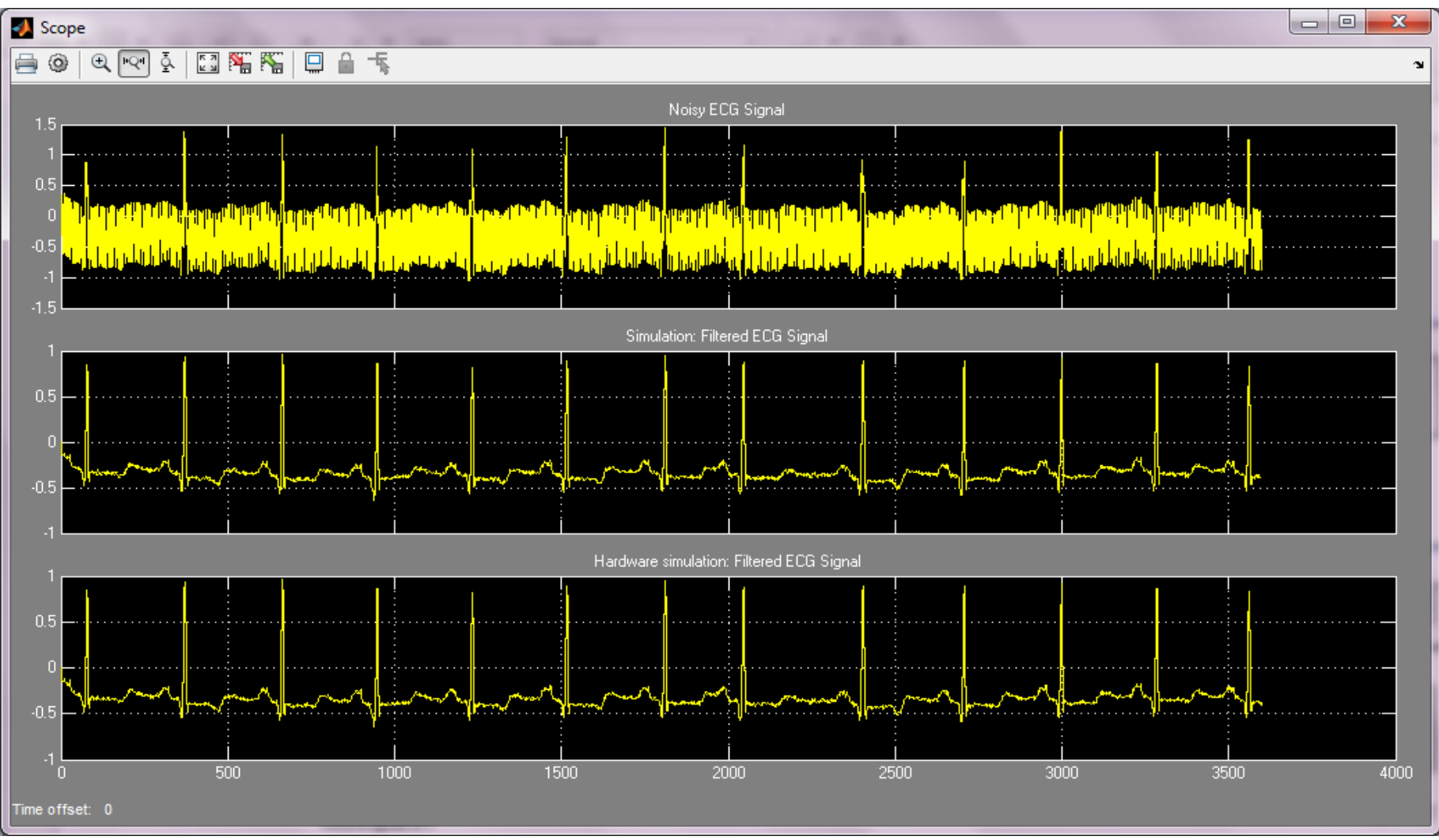

Fig-8: Simulation and Hardware cosimulation results of Filtered ECG signal of Swarm algorithm based Adaptive filter. 




Fig-9: Wavescope result of Filtered ECG signal of Swarm algorithm based Adaptive filter.

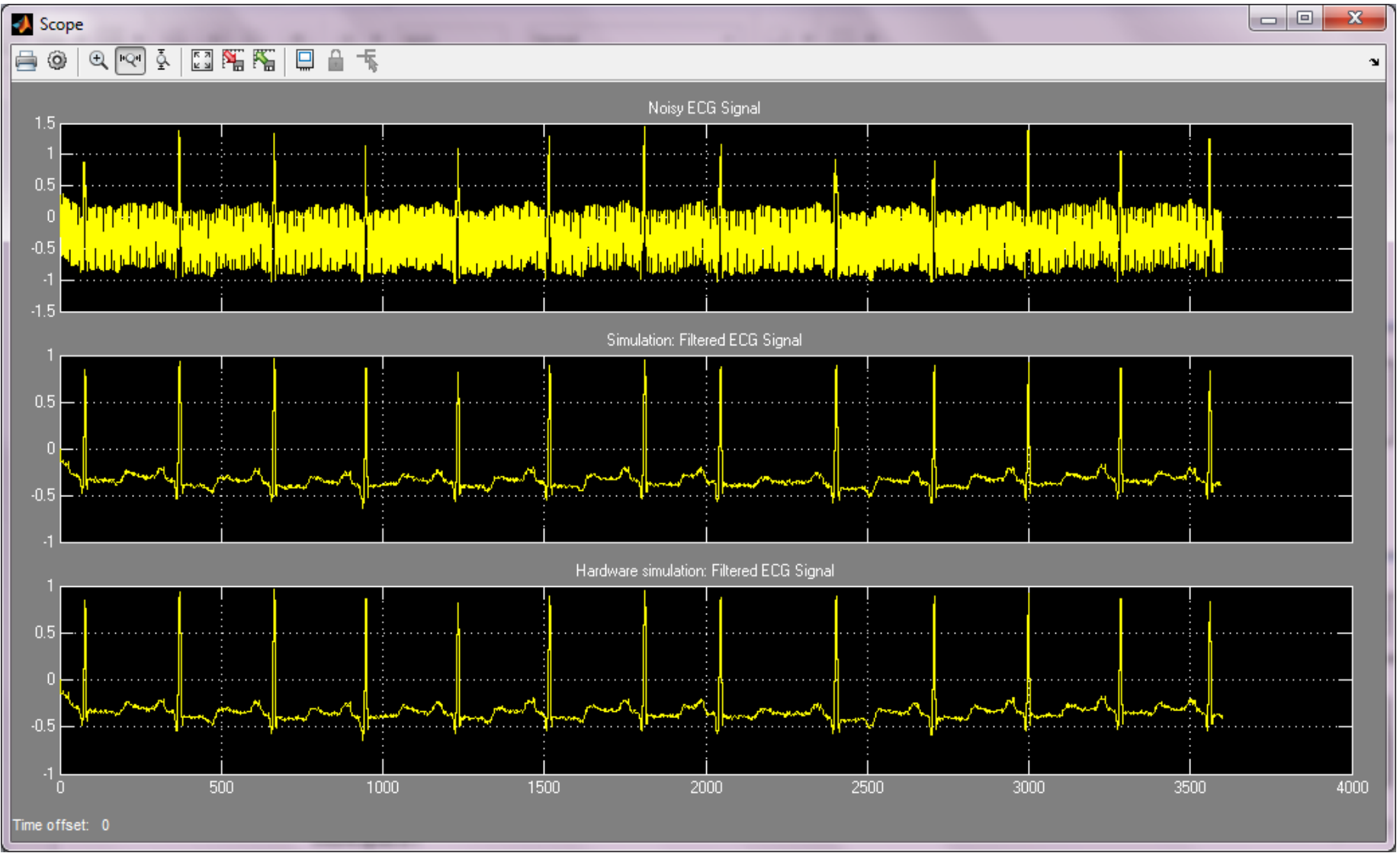

Fig-10: Simulation and Hardware cosimulation results of Filtered ECG signal of two stage Swarm algorithm based Adaptive filter. 


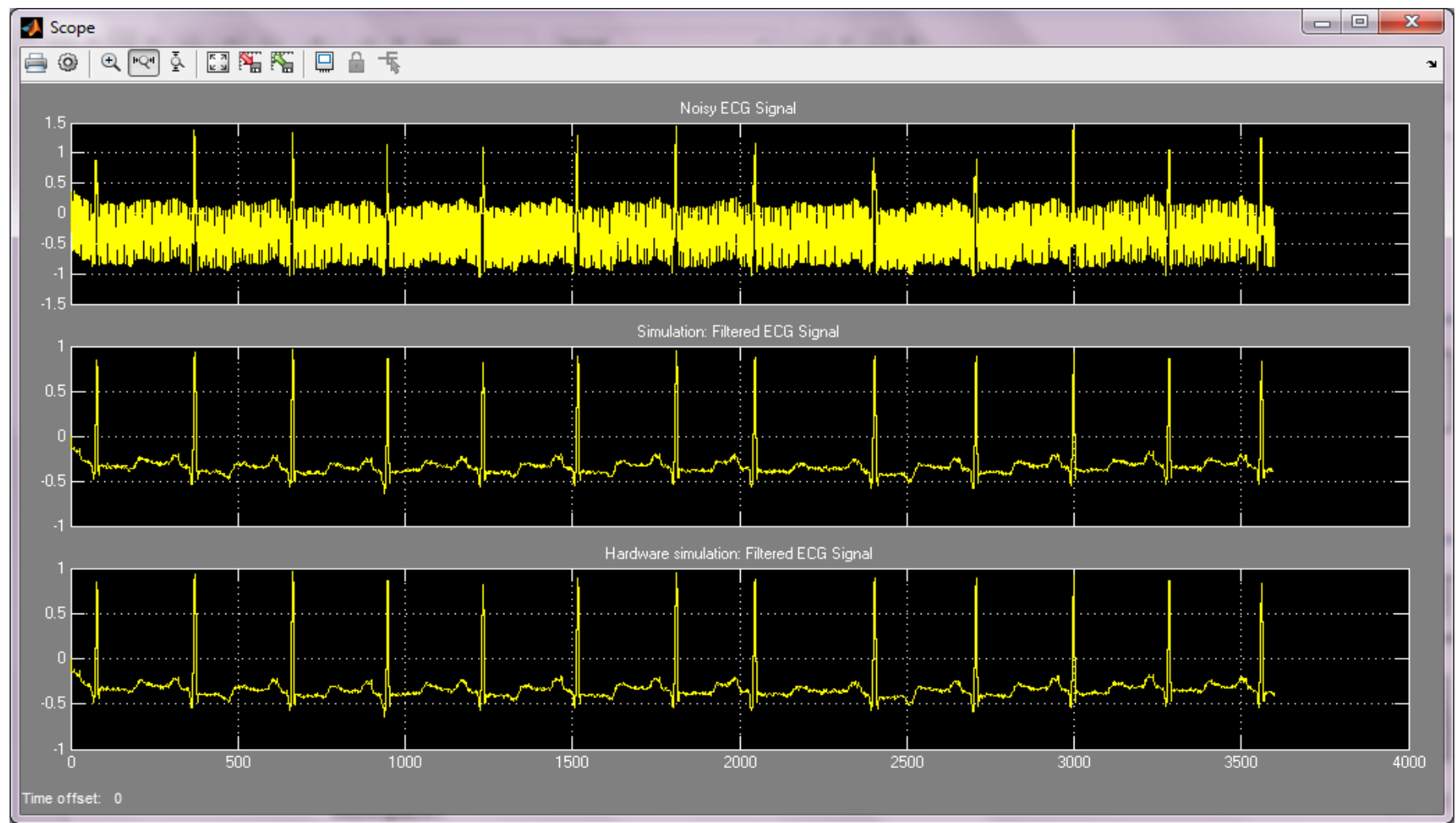

Fig-11: Simulation and Hardware cosimulation results of Filtered ECG signal of three stage Swarm algorithm based Adaptive filter.

\section{CONCLUSION}

This paper introduced newly adapted Swarm Optimization algorithm on Adaptive filter for removing Power Line Noise. This total system is implemented on Spartan-3 board for high speed performance. Swarm Optimization based Adaptive filter with single stage is sufficient for removing Power Line Noise from the ECG signal. Single stage adaptive filter performance is same as that of the multistage, hence it is not necessary to design multistage Adaptive filter. Adaptive filter based on Swarm optimization algorithm gives better speed and efficiency.

\section{ACKNOWLEDGEMENTS}

The Authors are thankful to Department of Technology, Shivaji University, Kolhapur, India for providing necessary facilities for completion of this manuscript

\section{REFERENCES}

[1]. R J. Mahil · T. Sree Renga Raja, "An intelligent biological inspired evolutionary lgorithmfor the suppression of incubator interference in premature infants ECG", Springer-Verlag Berlin Heidelberg 2013.

[2]. AcamporaG, Lee C-S,Vitiello A,WangM-H(2012) "Evaluating cardiac health through semantic soft computing techniques". Soft Comput 16(7):1165-1181.

[3]. Beligiannis GN, Skarlas LV, Likothanassis SD, “ Perdikouri D (2005) Nonlinear model structure identification of complex biomedical data using a genetic programming based technique". IEEE Trans Instrum Meas 54(6):2184-2190.
[4]. Chang C-Y, Chen D-R (2010), “Active noise cancellation without secondary path identification by using an adaptive Genetic algorithm". IEEE Trans Instr Meas 59(9):2315-2327.

[5]. Y. Kumar and G.K. Malik, "Performance Analysis of different Filters for Power Line Interface Reduction in ECG Signal", In International Journal of Computer Applications (0975 - 8887), vol. 3 -No.7, pp.1-6, June 2010.

[6]. Lin YD, Hu YH (2008), "Powerline interference detection and suppression in ECG signal processing". IEEE Trans Biomedical Engineering 55(1):354-357.

[7]. Suzanna M. M. Martens and Jan W. M. Bergmans, "An Improved Adaptive Power Line Interference Canceller for Electrocardiography" IEEE Transaction on Biomedical Engineering, VOL. 53, NO. 11, November 2006

[8]. Aarti J. Patel, "Nyquist Filter Design to Remove Power Line Interference From Bio-Electric Signals" International Journal of Engineering Trends and TechnologyVolume3Issue2- 2012 ISSN: 2231-5381.

[9]. Lin YD, Hu YH (2008), "Powerline interference detection and suppression in ECG signal processing”. IEEE Trans Biomedical Engineering 55(1):354-357.

[10]. Martennns SMM, Mishi M, Oei SG (2006), “An improved adaptive powerline interference canceller for electrocardiography". IEEE Trans Biomed Eng 53(11):2220-2231.

[11]. Rout NK, Das DP, Panda G (2012), "Particle swarm optimization based active noise control algorithm without secondary path identification". IEEE Trans Instrum Meas 61(2):554-563. 
[12]. Thakar NV, Zhu YS (1998), “Applications of adaptive filtering to ECG analysis: noise cancellation and arrhythmia detection". IEEE Trans Biomed Eng 38(8):785-794.

[13]. Ziarani AK (2002), "A nonlinear adaptive method of elimination of powerline interference in ECG signals", IEEE Trans Biomed Eng 49(6):540-547.

[14]. Ying-Wen Bai, Wen-Yang Chu, Chien-Yu Chen, YiTing Lee, Yi-Ching Tsai and Cheng-Hung Tsai, "Adjustable $60 \mathrm{~Hz}$ Noise Reduction by a Notch Filter for ECG Signals, "Proceedings of the Instrumentation and easurement 'technology Conference Como. Italy.18-20 May 2004

\section{BIOGRAPHIES}

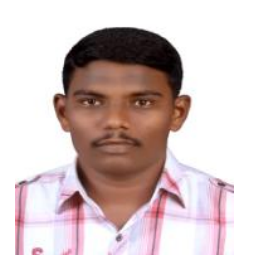

M.TECH Scholar, Electronics Department, Department of Technology, Shivaji University, Kolhapur. India (MS). galphadevikram083@gmail.com.

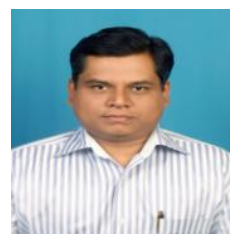

Assistant Professor, Pursuing Ph.D, Electronics Department, Department of Technology, Shivaji University, Kolhapur. India (MS).

pxbhaskar@yahoo.co.in. 